\title{
Scientific Abstracts from the Winners of unERD 2018
}

\section{Inhibiting Apoptosis following Ischemic Stroke to Promote Neural Repair}

Roya Akbary ${ }^{1}$, Rehnuma Islam ${ }^{2}$ and Cindi M. Morshead ${ }^{1,2,3}$

1. Institute of Biomaterials and Biomedical Engineering, University of Toronto

2. Institute of Medical Science, University of Toronto

3. Division of Anatomy, Department of Surgery, Faculty of Medicine, University of Toronto

Stroke is one of the leading causes of death and disability in Canada with current treatment yielding poor outcomes. In ischemic stroke, a focal occlusion of blood flow causes necrotic cell death at the infarct site and hypoxia induced apoptosis (programmed cell death) in the perilesional brain region. Apoptosis is initiated by caspase enzymes and therefore, caspase inhibitors could provide a means to promote cell survival. Previously explored caspase inhibitors have led to adverse effects due to non-specific inhibition. Herein we tested a proprietary selective caspase-3/7 inhibitor, which we hypothesize will improve cell survival at the stroke lesion site and reduce motor deficits after stroke. To test this hypothesis, young adult mice received ischemic stroke of the motor cortex and were injected daily with apoptosis inhibitor for either 4 or 8 days. In untreated, stroke injured mice, cell death within the perilesional area is complete by 4 days and the size of the lesion does not increase over time. Our preliminary histological findings, using cresyl violet, reveal that 4 and 8-day drug treatment results in a smaller lesion area and volume compared to untreated stroke mice. Neurons and astrocytes are currently being analyzed and we predict more neurons will be found in drug treated mice. With the goal of improving functional recovery, we are examining the effects of drug treatment on motor impairment following stroke. Our improved understanding of the potentially neuroprotective effects of caspase inhibitors will contribute to the development of much-needed novel therapies for stroke.

\section{Understanding the Relationship of S-Palmitoylation, Autophagy and Apoptosis}

Bilal Hussain ${ }^{1}$, Haisam Shah ${ }^{1,2}$ and Scott Heximer ${ }^{1,2}$

1. Institute of Biomaterials and Engineering, University of Toronto

2. Ted Rogers Centre for Heart Research
Autophagy is a lysosomal pathway involved in the degradation and recycling of damaged cellular proteins and organelles [1]. Protein S-palmitoylation is the post-translational modification of proteins where palmitic acid, a 16-carbon long saturated fatty acid, gets covalently attached to the cysteine side chain of a protein [2]. Specialized transmembrane proteins called palmitoyl acteyl-transferases (DHHC) are the sites for s-palmitoylation. Both autophagy and palmitoylation are vital cellular processes that have been implicated in the pathogenesis of neurodegenerative and cardiovascular diseases. However, the molecular and regulatory relationship of s-palmitoylation and autophagy remains to be poorly understood. Using Human Embryonic Kidney (HEK) cells our lab showed that the pharmacological inhibition of palmitoylation with 2-bromopalmitate (2-BP) and the induction of autophagy with rapamycin resulted in apoptosis. We are interested in further investigating the molecular mechanisms involved in 2-BP and Rapamycin induced apoptosis. We treated HEK cells with 2-BP and rapamycin, along with different autophagy inhibitors 3MA, Bafilomycin A1 and Leupeptin to determine whether the inhibition of autophagy could prevent cells from undergoing apoptosis. Second, we transfected HEK cells with inactive palmitoyl acetyl-transferases (DHHS) 3,5,7,21 to investigate, which of the DHHC enzymes are necessary for cell death. Our results show that 3MA-reduced rapamycin - 2-BP induced apoptosis, while Bafilomycin A1 and Leupeptin did not. Furthermore, the inhibition of palmitoyl acetyl-transferases 3,5,7, and 21 did not result in apoptosis when treated with rapamycin. From our current data we have identified a novel relationship between palmitoylation, autophagy and cellular survival. However further work is necessary in characterizing the molecular mechanisms involved.

\section{Material Property Analysis of Graphene-Concrete Composites}

Michael McLean ${ }^{1}$, Tanvir Qureshi², Daman K. Panesar, ${ }^{3}$ Department of Civil Engineering, University of Toronto

The cement industry is responsible for approximately $5 \%$ of global carbon dioxide emissions [1], and cement production is a major contributor to greenhouse gas (GHG) emissions in the construction industry. Further, in 2015, Environment and Climate Change Canada reported that cement and concrete production accounts for $14 \%$ of the carbon dioxide emitted by Canada's manufacturing industry [2]. These facts display the urgency for which the impact of cement production on the atmosphere must be reduced. The aim of this research project is to measure and analyze the material properties of a set of graphene-concrete composites, 
to show their potential as stronger, more durable, "green" alternatives to general use concrete. The three graphene materials used are pure graphene, graphene oxide, and reduced graphene oxide. Each was dispersed within water, which was then used as part of a standard mix design to make four concrete cylinders per batch. Three cylinders per batch were compression tested for their maximum compressive strength, and the last cylinder was used in the rapid chloride ion penetration test (RCPT). Compressive strength and resistance to chloride ion penetration (indicating corrosion resistance) were measured and compared to a general use concrete control group. Results thus far have indicated that some of these composites possess a higher compressive strength which allows for a lower volume of cement to be used in any given application. Further, they generally possess higher durability and thus a longer lifespan than general use concrete, which has potential to reduce the amount of cement needed for structure maintenance.

\section{Analysis and Modeling of GTHA Public Transit Fare Integration}

Patrick Meredith-Karam ${ }^{1}$, Eric J. Miller, Ph.D. ${ }^{2,3}$

1. Division of Engineering Science, University of Toronto

2. University of Toronto Transportation Research Institute, University of Toronto

3. Travel Modeling Group, University of Toronto

In collaboration with the Travel Modeling Group (TMG) within the University of Toronto Transportation Research Institute (UTTRI), this project aims to develop transit fare modeling capabilities in TMG's GTAModel. The model is an operational travel demand forecasting system used by several Greater Toronto-Hamilton Area (GTHA) municipalities for transportation policy analyses. A lack of integrated fare policy is a challenge facing transit agencies across the GTHA. Many trip-makers traverse multiple agencies, and the lack of an integrated fare system is a barrier to seamless travel. Metrolinx has identified the need for integrated fare policy, and several options have been proposed by their Chief Planning Officer [1]. Accurate fare modeling will be essential to evaluate these policy options. The project first involved a review of literature and fare policies to identify the current and proposed future states of GTHA fare integration. Secondly, a comprehensive evaluation was performed to determine the suitability of AgentBased Microsimulation methods (particularly MATSim software) for future GTAModel releases. Finally, research was performed to compile accurate and comprehensive GTHA fare policy information, as an input to GTAModel's V4.1 release. Methods used include literature review and research on software documentation, as well as fare policy research and outreach to transit agencies. Upon completion of the project, implementation of the fare information inputs and chosen fare modeling procedure for GTAModel were evaluated based on their ability to accurately represent fare prices for current and potential future fare policies.

\section{Sustained release of a thermostabilized mutant of Chondroitinase $\mathrm{ABC}$ for treatment of spinal cord injury}

Andrew J. Pickering ${ }^{1}$, Marian H. Hettiaratchi ${ }^{1}$, Carter J. Teal ${ }^{2}$, Matthew J. O'Meara ${ }^{3}$, Brian K. Shoichet ${ }^{3}$, Molly S. Shoichet ${ }^{1,2,4}$ 1. Department of Chemical Engineering and Applied Chemistry, University of Toronto

2. Institute of Biomaterials and Biomedical Engineering, University of Toronto

3. Department of Pharmaceutical Chemistry, University of California, San Francisco

Department of Chemistry, University of Toronto

Spinal cord injury (SCI) is a debilitating condition that affects over 85000 Canadians [1]. Functional recovery is limited by the formation of a glial scar, which creates an inhibitory environment that prevents tissue repair [2]. Chondroitinase $\mathrm{ABC}$ (ChABC) is a bacterial enzyme that degrades the glial scar, and has been shown to be an effective treatment in preclinical models of SCI [3]. However, repeated intraspinal injections of ChABC are highly invasive, and sustained release formulations have shown modest efficacy due to its thermal instability [4]. We hypothesize that a thermostabilized ChABC mutant delivered in a methylcellulose hydrogel will allow for a sustained release of bioactive ChABC. Computational modelling was used to design three point mutations in ChABC by minimizing the predicted change in folding free energy [5]. Mutants were screened for expression and yield in two different strains of E. Coli, and purified using a nickel affinity column and size exclusion chromatography. Protein expression was confirmed using gel electrophoresis, and activity was evaluated at $37 \mathrm{oC}$ by measuring chondroitin-sulphate A degradation. Preliminary results demonstrated that one of the mutants was expressed with five-fold greater yield, and increased the half-life to 17.5 hours, compared to only 7.8 hours with the native protein. Ongoing experiments are investigating the release of this $\mathrm{ChABC}$ mutant from a methylcellulose hydrogel. The optimized formulation will eventually be tested in a rat model of spinal cord injury and has the potential to significantly extend the therapeutic effect of $\mathrm{ChABC}$ and improve functional recovery. 